\title{
Surface inspection problems in thermoelectric testing
}

\author{
Ahmed Abouellail ${ }^{1, *}$, Igor Obach $^{2}$, Andrey Soldatov ${ }^{1}$, and Alexey Soldatov ${ }^{1}$ \\ ${ }^{1}$ Tomsk Polytechnic University, 634050 Tomsk, Russia \\ ${ }^{2}$ Tomsk State University of Control Systems and Radioelectronics, 634050 Tomsk, Russia
}

\begin{abstract}
This paper demonstrates the outcome of experimental studies on thermoelectric characteristics of thermocouples. Measurements were carried out using different types of thermocouples; each studied alone then investigated when they are connected in parallel, in order to simulate the imperfect nature of the various multi-contact surface of the tested object. The investigated types were Chromel-Alumel and Nichrome-Constantan. The thermoelectric characteristics were measured at the temperature range $160^{\circ}-400^{\circ}$ Celsius, in order to identify the best operating temperature for the hot electrode. Furthermore, the load resistance is another important factor that has been investigated and therefore varied from $1 \Omega$ to $10 \mathrm{k} \Omega$, in order to determine its effect on the electrical characteristics of thermocouples. Accordingly, these characteristics will help defining the requirements for an optimum thermoelectric testing.
\end{abstract}

\section{Introduction}

The thermoelectric testing method is widely used in non-destructive testing of metals and alloys [1-3]. However, unreliable results are often obtained due to the heterogeneity and roughness of the surface, which correspondingly leads to a multipoint contact $[4,5]$. The impact of the surface quality has been investigated and the results are presented later in this paper. Multipoint contact results in equivalent circuits of parallel connection of several thermocouples that have different characteristics, due to local fluctuations of the chemical composition of the metal or alloy $[6,7]$.

In order to take into account the impact of the resulted thermocouples on the inspection results, it is necessary to study their characteristics. According to the carried out literature reviews, the study of thermocouples' characteristics has not been conducted until today. Taking account of these characteristics and the degree of influence of the above factors will significantly improve the technical characteristics of the thermoelectric inspection devices.

\section{Experimental investigation}

In this study, the characteristics of different parallel thermocouples has been investigated in the range of $160^{\circ} \mathrm{C}-400^{\circ} \mathrm{C}$. Characteristics of the thermocouples, Chromel-Alumel and

\footnotetext{
* Corresponding author: demo 092@icloud.com
} 
Nichrome-Constantan have been investigated, when each is alone and when both are connected in parallel.

A block diagram of the experimental setup is shown in Figure 1(a) and it includes:

1) A heat chamber, $T$;

2) Thermocouples of different types, each consists of hot and cold electrodes;

3) Load Resistance, $\mathrm{R}_{\mathrm{L}}$ (of nominal values: $1 \Omega, 5.2 \Omega, 10 \Omega, 100 \Omega, 1 \mathrm{k} \Omega, 10 \mathrm{k} \Omega$ );

4) Measuring instruments (voltmeter and ammeter).

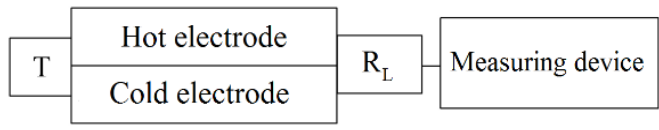

(a)

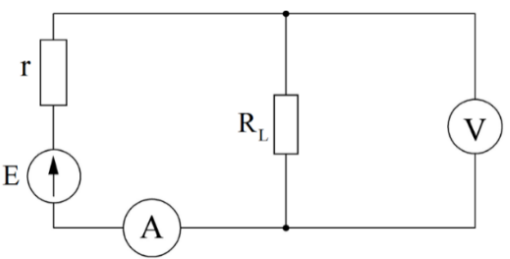

(b)

Fig. 1. The experimental setup (a) Block diagram (b) Schematic diagram, where $\mathrm{E}$ is the thermoelectric power source, $r$ is internal resistance and $R_{L}$ is the load resistance.

The thermocouple is presented in the schematic diagram of the experimental setup, which is shown in Figure 1(b), as a voltage source $E$ with an internal resistance $r$. The thermocouples Nichrome-Constantan and Chromel-Alumel were alternately put in a heat chamber at a varied temperature from $160^{\circ} \mathrm{C}$ to $400^{\circ} \mathrm{C}$. The voltage drop across the load resistance $\mathrm{R}_{\mathrm{L}}$ was carefully measured.

Measurements were performed in single and parallel connections of thermocouples, along with the load resistance $\mathrm{R}_{\mathrm{L}}$. Calculations of the thermoelectric power, the internal resistance $\mathrm{r}$, the flowing current in the circuit I, and the maximum electric power $\mathrm{P}$, were all carried out later according to the formulas shown below.

\subsection{Calculation of the thermocouple internal resistance, $r$}

According to the second Kirchhoff's law [8], the value of EMF in the circuit is equal to the sum of the voltage drops across the resistors. This means:

$$
E=V_{r}+V_{L}=I r+I R_{L}
$$

where $\mathrm{E}$ is the thermoelectric power, $\mathrm{V}_{\mathrm{r}}$ is the voltage drop across the internal resistance $\mathrm{r}$, $V_{L}$ is the load voltage, $R_{L}$ is the load resistance and $I$ is the current flowing across $R_{L}$.

By manipulating expression (1), we obtain:

$$
r=\frac{E-I R_{L}}{I}
$$

However, E is considered maximum under the condition of open-circuit test:

$$
E=V_{\max }=V_{O C}
$$

where $E_{\max }$ is the maximum measured thermoelectric power obtained and $\mathrm{V}_{\mathrm{OC}}$ is opencircuit voltage load. 


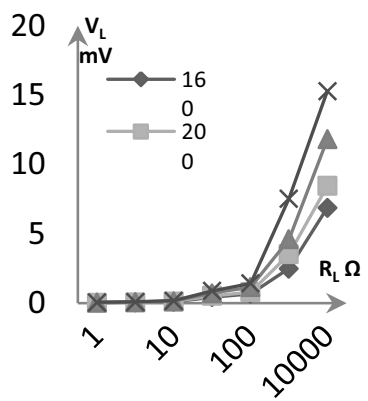

(a)

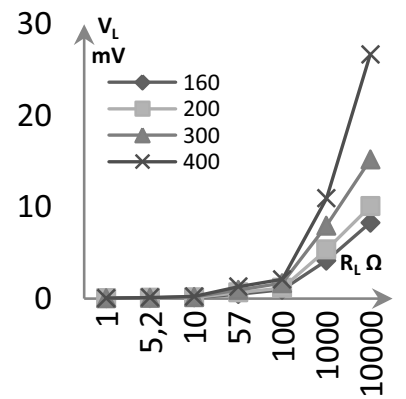

(b)

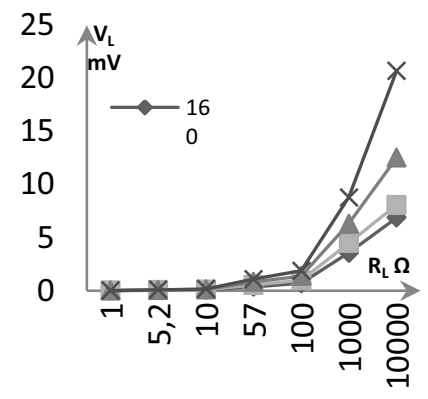

(c)

Fig. 2. The dependence curves of the load voltage on the load resistance, where each curve represents a different temperature $\left(160^{\circ} \mathrm{C}, 200^{\circ} \mathrm{C}, 300^{\circ} \mathrm{C}, 400^{\circ} \mathrm{C}\right.$ ). (a) Chromel-Alumel, (b) NichromeConstantan, (c) Chromel-Alumel in parallel connection with Nichrome-Constantan.

The results of the voltage measured across the load, with each temperature condition, are presented in Figure 2 as it shows the investigated characteristics of Chromel-Alumel, Nichrome-Constantan and the case for their connection together in parallel.

As shown in the graphs, as the load resistance increases $R_{L}$, the load voltage $V_{L}$ also increases, until it approaches the maximum value of thermoelectric power, at which the open-circuit test is carried out. Upon the increase in temperature, the value of $\mathrm{V}_{\mathrm{L}}$ increases as well, due to the increase of thermoelectric power, which is proportional to temperature.

The characteristics of the calculated thermocouple internal resistance have been also investigated and it was found out that as the load resistance increases, the value of the internal resistance increases proportionally. Moreover, it has been observed that the internal resistance, in case of parallel connection of thermocouples, is an equivalent average value of both internal resistance curves of each single thermocouple when investigated alone.

\subsection{Calculation of the electric power of thermocouples, $\mathrm{P}$ and $\mathrm{P}_{\max }$}

Power is determined by the expression [9]:

$$
P=I^{2} R_{L}
$$

where P stands for power.

From Figure 1(b) we find the current in the circuit: $I=\frac{E}{R_{L}+r}$

By substituting the values from formula (4) into formula (5), we have:

$$
P=\frac{E^{2} \times R_{L}}{\left(R_{L}+r\right)^{2}}
$$

To find the global maximum point, we take the derivative of $P$ with respect to $R_{L}$

$$
\frac{d P}{d R_{L}}=\frac{E^{2}\left(R_{L}+r\right)^{2}-2 E^{2} R_{L}\left(R_{L}+r\right)}{\left(R_{L}+r\right)^{4}}
$$

After the unification of the general terms we get:

$$
\frac{d P}{d R_{L}}=E^{2} \frac{\left(R_{L}^{2}-r^{2}\right)}{\left(R_{L}+r\right)^{4}}
$$

$\mathrm{E}$ is never be equal to 0 and $\mathrm{r}$ can never be negative. This means the only left solution is:

$$
\left(R_{L}^{2}-r^{2}\right)=0
$$


This means the absolute maximum value of $\mathrm{P}$ is always at $r=R_{L}$. And as shown in Figure 3, the experimental results are confirmed by the calculations presented above. The maximum electric power is consistently observed when $r=R_{L}$.

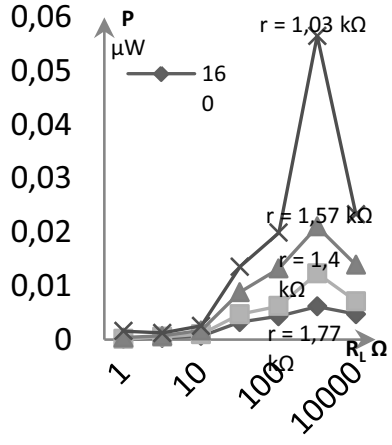

(a)

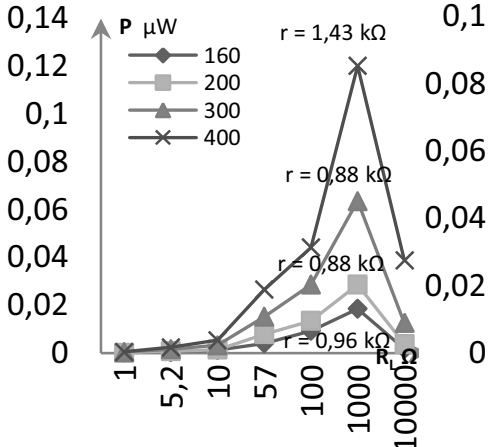

(b)

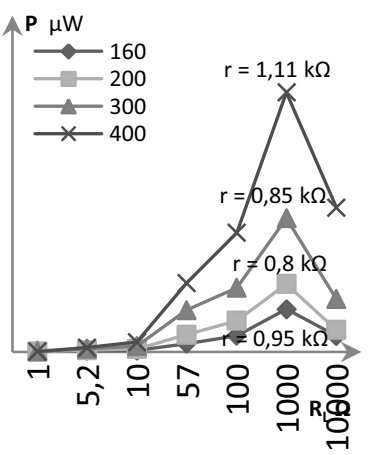

(c)

Fig. 3. The thermocouple power versus load resistance at temperatures $(160-400)^{\circ} \mathrm{C}$ (a) ChromelAlumel, (b) Nichrome-Constantan, (c) Their parallel connection.

\section{Conclusions}

Within this article, an investigation of the electrical characteristics of two thermocouples in parallel connection was conducted, in order to simulate and study the effect of multi-point contact between the sensing thermocouple electrodes and the inspected sample in thermoelectric inspection devices.

It has become clear from the conducted research that thermocouples, when connected in parallel, values of the internal resistance, electric power and thermoelectric power, are steadily equivalent to the resulted average values from thermocouples Chromel-Alumel and Nichrome-Constantan when each connected alone.

It has also been found experimentally that the most optimal load resistance for thermocouples is $1 \mathrm{k} \Omega$. This resistance satisfies the condition $r=R_{L}$, and consequently when thermocouple electric power is greatest. The choice of this load resistance would improve the accuracy of measurement of the thermoelectric inspection devices.

\section{References}

1. C. Stuart, J Test Eval 15, 224 (1987)

2. P. E. Mix, Introduction to Nondestructive Testing (John Wiley \& Sons, New Jersey, 2005)

3. A.I. Soldatov et al., SIBCON, 7491869 (2016)

4. A.A. Soldatov et al., RUSS J NONDESTRUCT+ 48, 184 (2015)

5. A.I. Soldatov et al., KEY ENG MAT 685, 310 (2016)

6. A.I. Soldatov et al., MEACS, 7414922 (2015)

7. J. Hu, P.B. Nagy, Appl Phys Lett 73, 467 (1998)

8. T.H. Davies, Mech Mach Theory 16, 171 (1981)

9. T. Croft et al., American Electricians' Handbook (McGraw-Hill, New York, 2009) 